\title{
Correlation Between Hormonal and Neurochemical Changes and Depression With Menopausal STATUS:A Systematic Review
}

\author{
Mostafa Chashmposh ${ }^{1}$; Saeed Shirali ${ }^{2, *}$; Esmaeel Ebrahimi ${ }^{1}$; Alireza Barari $^{3}$ \\ ${ }_{1}^{1}$ Department of Biochemistry, Faculty of Medicine, Ahvaz Jundishapur University of Medical Sciences, Ahvaz, IR Iran \\ 2 Department of Laboratory Sciences, School of Paramedical Sciences, Ahvaz Jundishapur University of Medical Sciences, Ahvaz, IR Iran \\ 3 Department of Physical Education and Sport Sciences, Science and Research Branch, Islamic Azad University of Ayatollah Amoli, Amol, IR Iran \\ *Corresponding author: Saeed Shirali, Department of Laboratory Sciences, School of Paramedical Sciences, Ahvaz Jundishapur University of Medical Sciences, Ahvaz, IR Iran. Tel: \\ +98-6113738319, Fax: +98-6113738330, E-mail: saeed.shirali@gmail.com
}

Received: October 31, 2014; Accepted: April 20, 2015

\begin{abstract}
Context: Menopause is characterized by amenorrhea and increase in the level of Follicular Stimulating Hormone (FSH) due to the permanent cessation of ovarian function. This process causes changes in hormonal and other serum markers. Depression is the leading cause of disease-related disabilities in women.

Objectives: The aim of this review was to investigate the correlation between hormonal and petrochemical changes and depression with menopausal status.

Data Sources: We searched in Cochrane Library, MEDLINE, PubMed, Google Scholar, Web of Science and Scopus, Embase, the reference lists of all related studies and major relevant review articles from 1960 to October 2014, and also abstracts from associated congresses and meetings, using terms related to hormonal and serum markers, depression and its symptoms.

Study Selection: The survey included prospective, retrospective and case-control studies. The selected studies explored menopause in study population and investigated variables and different markers in depression or anxiety, as well as those measuring depression or anxiety intensity.

Data Extraction: Items for which data were extracted included the date and place of publication, study design, sources, human species, age, control groups, selection and appraisal methods, outcome measurement tools, and author's conclusions. One investigator (Mostafa Chashmposh) collected the relevant reports, whereas two other authors independently reviewed the published data and reported different hormonal markers related to depression as evaluated by different studies. Disagreements were resolved by the fourth reviewer's decision.

Results: The period of menopause is mostly associated with a gradual decline of estrogen activity and increased secretion of nocturnal melatonin. During menopausal transition, overnight cortisol levels were associated with changes in estrone glucuronide, testosterone, and FSH levels. In addition, whole blood serotonin concentration is reduced during menopause. Furthermore, lipid markers including total and LDL cholesterol levels increase during perimenopause. Moreover, plasma Brain Derived Neurotropic Factor(BDNF)concentration decreases significantly in postmenopausal period. Based on different studies, the reduction of hormones including estrogen, serotonin and BDNF during menopause are associated with depression in women. Further studies documented the relationship between depressed mood symptoms and cortisol levels.

Conclusions: The available evidence suggests that transition to menopause and its changing hormonal and other serum markers are strongly associated with depressed mood among women.
\end{abstract}

Keywords: Menopause; Depression; Hormonal Changes; Neurochemical Changes

\section{Context}

Menopause, the final menstrual stage, is defined as at least 12 months of amenorrhea and increase in serum Follicle-Stimulating Hormone (FSH) and decreases in estradiol (1). The average age at menopause is approximately 51 years (2). Menopause process causes hormonal changes accompanied by decreasing levels of sex hormones including estrogens and androgens. Similar changes are induced by ovarian malfunction created by cancer and surgical menopause. Changes in hormonal and serum markers can affect many tissues and generate a variety of symptoms, including vasomotor signs, osteoporosis, imbalance in energy metabolism and mood, pubic hair loss and atrophy of gonads and sexual malfunction (3). Hormonal changes take place in three stages including pre, peri and post menopause. In Premenopause stage, the levels of reproductive hormones become more irregular and hormone withdrawal symptoms begin to take effect (4). Next stage, perimenopause, is of unknown features and occurs 2-8 years earlier than the last menses. In early perimenopause, neurohormonal systems begin to become deregulated, without alteration in cycle length. The middle and the end of perimenopause are characterized by irregular menstrual cycles, especially by short cycles associated with longer intervals between cycles (2). Post-

Copyright (C) 2015, Health Policy Research Center, Shiraz University of Medical Sciences. This is an open-access article distributed under the terms of the Creative Commons Attribution-NonCommercial 4.0 International License (http://creativecommons.org/licenses/by-nc/4.0/) which permits copy and redistribute the material just in noncommercial usages, provided the original work is properly cited. 
menopausal stage involves women without menstrual cycle for at least 12 months and incapable of becoming pregnant or having lactation (4). Mood disturbances including depression will lead to an epidemic proportion in 21st century worldwide (5). Depression is the main reason for disease related inability in women. Different researches have revealed that the prevalence of a major depressive disorder in women is approximately twice higher than that of men (6). This prevalence could create many problems and expenses for various nations. Different biologic markers can be used to evaluate depressive states. Endogenous depressions are multifactorial, where the main non-endocrine risk factors for depressive syndromes are 'empty nest', daily stress, health problems and death of family members or a close friend. Other non-endocrine risk factors are ageing, the socio-economic situation and recent periods of stress. These risk factors occur frequently in people aged from 45 to 55 years, the age of the menopausal transition. On the other hand, it is well known that physiologically unstable endocrine periods in life such as puberty, pregnancy, the post-partum period, the premenstrual phase or the climacteric stage are endocrine high-risk situations, independent of non-endocrine risk factors (7). Transition to menopause has long been considered a period of increased risk for depressive symptoms. However, the extent to which this period represents increased risk for major depressive disorder is less clear (8). In line with our recent studies on women's health, including diabetes (9) and depression (10), the present survey attempts to determine correlation between hormonal and neurochemical changes and depression with menopausal status. Additional aspect studied included treatment strategies reported in previous studies, which may improve women's health especially in relation to physiology and nutrition.

\section{Objectives}

The aim of this review was to investigate the correlation between hormonal and petrochemical changes and depression with menopausal status.

\section{Data Sources}

Studies selected were based on a systematic search in Cochrane Library, MEDLINE, PubMed, Google Scholar, Web of Science and Scopus, Embase and the reference lists of all relevant studies and major related review papers from April 1960 to October 2014, using terms in connection with hormonal and serum markers, depression and its symptoms and also abstracts from related congresses and meetings.

\subsection{Search Strategy}

All relevant articles were found using the key words: "Menopause," "Premenopause," "Perimenopause," "Postmenopausal," “Depression," "Serotonin," “Melatonin," "Cortisol," and "Hormonal changes" in various combina- tions. Figure 1 illustrates the process of our search strategy and the number of studies.

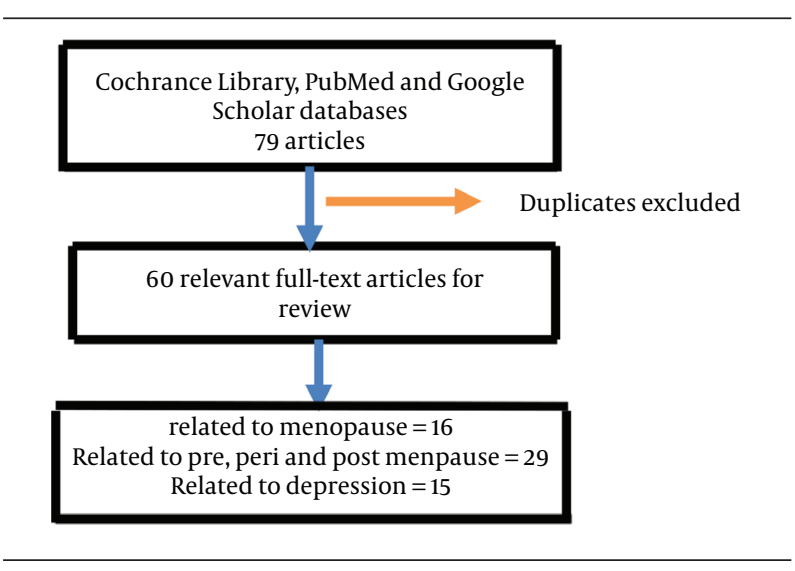

Figure 1. Flowchart of Study Selection for the Systematic Review

\section{Study Selection}

The survey included prospective, retrospective and case-control studies. The selected studies explored menopause in study population, investigated variables and different markers in depression or anxiety, as well as those measuring depression or anxiety intensity.

\section{Data Extraction}

Items for which data were extracted included the date and place of publication, study design, sources, human species, age, control groups, selection and appraisal methods, outcome measurement tools, and author's conclusions. One investigator (Mostafa Chashmposh) collected the relevant reports, whereas two other authors independently reviewed the published data and reported different hormonal markers related to depression as evaluated by different studies. Disagreements were resolved by the fourth reviewer's decision.

\subsection{Bias Prevention and Methodological Quality Assessment}

The quality assessment process was used for searching potential sources/risk of bias in each study. The methodological quality of trials was assessed independently using criteria from the Cochrane Handbook for Systematic Review of Interventions, Version 5.1.0 (W. Liu and X. J. Xiong) (11). The items included random sequence generation (selection bias), allocation concealment (selection bias), blinding of participants and personnel (performance bias), blinding of outcome assessment (detection bias), incomplete outcome data (attrition bias) and selective reporting (reporting bias).

\section{Results}

The results of previous studies have been classified into two groups; including hormonal and physiological 
changes during the menopausal transition and changes in serum markers of melatonin, cortisol, serotonin, brain derived neurotrophic factor or BDNF, lipids and lipoproteins during the menopausal transition. The summary of these results is shown in Table 1, but the results are fully presented in the text.

\subsection{Hormonal and Physiological Changes During the Menopausal Transition}

The period of menopause is mainly associated with an increase in the rate of depressive symptomatology characterized with slow decrease of estrogen activity. Therefore, the estrogen has been considered as the most important hormone concerned in the pathogenesis of menopause- related depression (12). Different studies involving estrogen therapy showed a mild improvement in depression in women at different phases of menopause $(19,20)$. However, this was not realized until Schmidt and colleagues in 1990 reported that estrogen treatment is better than placebo for perimenopausal depression (21). Pearlstein reported that rates of depression did not increase during the menopause but unstable mood and anxiety symptoms elevated years before menopause. In patients with cyclic affective disorders related to reproductive function, the depression risk is enhanced during the menopause (22). High levels of FSH were found in depressed and anxious patients (23). A study carried out on premenopausal women in 2003 demonstrated a high degree of interconnections between signs of depression and menopausal symptoms regarding clinical features and hormonal levels. Our data in this article support the relationship between depressive state, menopausal symptoms and different factors including sex hormones, cortisol, and serotonin deficiency (11). Decreased bone mineral density has been reported in patients suffering from several psychological disorders, such as schizophrenia, anorexia nervosa and major depressive disorders found in premenopausal women (24).

Table 1. Studies Exploring the Correlation Between Hormonal and Neurochemical Changes and Depression With Menopausal Status

\begin{tabular}{lcc}
\hline Research Groups & Study Subjects & Objectives \\
\hline Cohen LS et al.2006 (8) & $\begin{array}{c}\text { 460 premenopausal women, 36 to } 45 \\
\text { years of age, with no lifetime diagnosis } \\
\text { of major depression }\end{array}$ & $\begin{array}{c}\text { To examine the association between the } \\
\text { menopausal transition and onset of first lifetime } \\
\text { episode of depression among women with no } \\
\text { history of mood disturbance }\end{array}$
\end{tabular}

Rajewska J et al. To compare Results confirm the $2003(12)$

\begin{tabular}{|c|c|}
\hline & $\begin{array}{l}\text { year of age and } 30 \text { healthy control } \\
\text { women (mean age } 41 \text { years) }\end{array}$ \\
\hline $\begin{array}{l}\text { Okatani Y et al., To } \\
\text { evaluate therelation } \\
\text { between Nocturnal } \\
\text { melatonin secretion in } \\
1994(13)\end{array}$ & $\begin{array}{l}20 \text { women with SA, } 5 \text { women with } \\
\text { endometriosis showing normal men- } \\
\text { strual cycles and } 11 \text { volunteers with } \\
\text { normal menstrual cycles }\end{array}$ \\
\hline Okatani Y et al.1999 (14) & $\begin{array}{l}46 \text { premenopausal women, } 44 \\
\text { postmenopausal women, and } 11 \text { pre- } \\
\text { menopausal women with uterine leio- } \\
\text { myoma scheduled for hysterectomy } \\
\text { and bilateral salping-oophorectomy }\end{array}$ \\
\hline Woods NF et al. 2006 (15 & $\mathrm{N}=132$ women, up to 52 years \\
\hline
\end{tabular}

Woods NF et al.2006 (16) 169 women in the middle or late MT or early postmenopause stages

Gonzales GF et al.1993 (17) 31 regularly menstruating women aged 25 - 35 years, 10 premature ovarian failure women aged 20 - 38 years, 13 postmenopausal women aged $50-70$ years, 20 bilateral ovariectomised women aged 30 - 50 years

Sohrabji F et al. 1995 (18)
To compare the activity of gonadal hormones and serotonergic system in premenopausal women with or without depression in relation to clinical and hormonal menopause factors

To evaluate the relation between endogenous estrogen concentrations and nocturnal melatonin secretion occurring in patients with Secondary Amenorrhoea (SA)

To measure changes in nocturnal melatonin and to characterize the role played by estrogen in controlling nocturnal melatonin secretion in perimenopausal women

To examine the influence of menopause related factors, stress-related factors, symptoms, social, and health-related factors on cortisol levels during the menopausal transition

To determine whether cortisol levels change prospectively during the Menopausal Transition (MT)

To investigate the effect of ageing and reduction in ovarian function on whole blood serotonin levels and the effect of the estrogen replacement in postmenopausal women on blood serotonin levels

To study the role and mechanism of estrogen action in the survival and differentiation of neurons in the basal forebrain and its targets in the cerebral cortex, hippocampus, and olfactory bulb
Within a similarly aged population of women with no lifetime history of depression, those who enter the menopausal transition earlier have a significant risk for first onset of depression

Results confirm the association between depressive and menopausal symptoms as well as an involvement of gonadal hormones, cortisol, and serotonin deficiency in this process

Nocturnal melatonin secretion in women with secondary amenorrhoea may be related to their low oestrogen concentrations

Transient elevated nocturnal melatonin secretion during menopause may be related to the existence of a low estrogen environment

Overnight cortisol levels during the MT were associated with E1G, testosterone, and FSH levels.

Cortisol levels rise with age, but have not been linked to stages of the MT

Whole blood serotonin concentration is reduced during menopause by suppression of ovarian function but may also be an effect of ageing

Estrogen may regulate BDNF transcription 
Chashmposh M et al.

\subsection{Changes in hormones and serum markers dur- ing the menopausal transition}

\subsubsection{Melatonin}

Melatonin is produced in the pineal gland and its diurnal pattern is connected to circadian rhythm, with low level of production in day and high concentration at night (25). The adrenergic stimulation of N-Acetyltransferase (NAT) function at night is responsible for this diurnal difference (26). Because of detection of sex hormone receptors in the pineal gland of rat $(27,28)$, this data has supported the regulation of melatonin synthesis by the sex steroids $(29,30)$. The nocturnal secretion of melatonin is markedly increased in women with secondary amenorrhea whose estrogen levels are extremely low (31, 32); exogenous estrogen stimulation suppressed such melatonin secretion (13). However, Bartsch and coworkers have stated that both transdermal and oral estradiol therapy can enhance and also reduce melatonin synthesis in different postmenopausal women (33). Okatani et al. found a temporary increase in pineal melatonin synthesis during the perimenopausal period in rats. They also found that the increased melatonin synthesis may result from decreasing levels of endogenous estrogen during this period (14). In another study, Okatani Y et al. stated the role of estrogen in regulating nocturnal melatonin synthesis in perimenopausal women. They assayed the changes of nightly melatonin secretion in perimenopausal women and then investigated the effect of oophorectomy and estrogen therapy on nocturnal melatonin synthesis in these women (25).

\subsubsection{Cortisol}

Cortisol plays a key role in facilitating responses to psychobiological load (34). The diurnal pattern of cortisol is connected to circadian rhythm, eating and sleeping, physical actions, and challenges in life (15). Based on previous studies, we hypothesize that cortisol levels would raise with age $(35,36)$. Furthermore, different evidences would reinforce a hypothesis that cortisol concentration is increased in response to both enhanced endogenous and exogenous estrogen levels $(37,38)$. Past evidences documented a relationship between depressed mood symptoms and cortisol levels (39). Rising cortisol levels have been associated with poor health, including lower bone density in older women (40), and could be hypothesized to relate to perceptions of poor health (41). In a study, Woods $\mathrm{N}$ et al. observed an increase in overnight cortisol levels as women transitioned from the early to the late menopausal transition stage (16). In another study, Woods $\mathrm{N}$ et al. stated that the overnight cortisol concentration in menopausal transition was connected with estrone glucuronide, testosterone, and FSH concentrations. Furthermore, they were considerably associated with norepinephrine and epinephrine hormones (15).

\subsubsection{Serotonin}

Serotoninergic nerve cell bodies are placed in the raphe nuclei of the midbrain and have various functions all over the brain, containing regions implicated in emotion modulation and cognition like the limbic system, hippocampus and frontal cortex (42). During menopause, Serotonin level is decreased by halting ovarian function which may also be affected by ageing. Gonzales et al. stated that in ovariectomised/natural postmenopausal or Amenorrheic women, serum serotonin concentration is lower than regularly menstruating women and serum serotonin concentration enhanced after estrogen therapy in postmenopausal women. They indicated that in ovariectomised women, serum serotonin concentration were inversely associated with age. On the other hand, in women with different blood estradiol concentration, a direct association was found between blood estradiol and blood serotonin concentrations (17). Latest neurobiological studies reveal that serotonin is the neurotransmitter mainly concerned in the pathogenesis and improves the menopausal depression (11). This may be because of multiple interactions of serotonin system with estrogen (43). An abnormality of serotonergic system was shown in perimenopausal depressed women (44) and serotonin dysregulation was also involved in the pathogenesis of somatic menopausal signs like hot flushes, night sweats etc., (45). The majority of antidepressant drugs execute their therapeutic functions via serotonergic mechanism (11), which is associated with a regulatory effect of estrogen on serotonin receptors, including 5HT1A and 5HT2, as targets of antidepressant drugs, beside increasing the serotonergic function (46).

\subsubsection{Brain Derived Neurotrophic Factor or BDNF}

BDNF is mainly considered as neurotrophin inside the brain (47) and modulates growth and survival, morphological plasticity and finally synthesis of new neurons with differentiated function (48); moreover, BDNF is considered to regulate other neurotransmitters including monoamines, amino acids, and peptide like serotonin and dopamine (49). In preceding research, Begliuomini et al. revealed that blood BDNF alterations are directly associated with those of estradiol and progesterone over the entire menstrual cycle. Also, the blood BDNF level reduces significantly in postmenopausal stage, supporting a major role of gonadal hormones in the modulation of neurotrophin expression (50). Therefore, the physiopathology of climacteric signs including depression, hot flashes etc., may correlate with the wide spectrum of sex hormones actions on neurons functions both directly through hormone receptor relations and indirectly via alterations in neurotransmitter concentration and neurotrophin activity (51). Some reports suggesting the involvement of BDNF in regulating estrogen actions showed that it could modulate the expression of BDNF via the estrogen response element on the BDNF gene (18, 
52). In recent years, the importance of non-hormonal alternatives for the decreasing the climacteric symptoms has enhanced, i.e. the use of Selective Serotonin Reuptake Inhibitors (SSRIs) for the treatment of vasomotor and neuropsychiatric diseases. Paroxetine, the most important of SSRIs is shown to considerably decrease hot flush frequency/severity composite scores in trials $(53,54)$, in that paroxetine improves the depressive state by increasing BDNF levels.

\subsubsection{Lipids and Lipoproteins}

Epidemiological evidences show an inverse relationship between the consumption of $\omega$-3 long-chain polyunsaturated fatty acids and depression incidence (55). Women in Premenopausal state have markedly less arteriosclerotic vascular disease than men. After menopause state, cardiovascular disease suddenly increases, with no difference between men and women aged more than 70 years. Hence, menopause is thought to be one of the risk factors for increasing lipids and cardiovascular diseases (56). Several studies have revealed that total cholesterol and LDL levels enhance after menopause $(57,58)$. Cho et al. reported the rising levels of LDL and total cholesterol levels in perimenopause, where changes in cholesterol are largely based on variations in the female gonadal hormones (59). These results suggest that changes in total cholesterol and LDL cholesterol and lipids associated with menopause are mainly dependent on female gonadal hormones. Furthermore, these outcomes suggest that depression is caused by increasing lipids during menopause. Nevertheless, a number of observational researches demonstrated that decrease in the levels of serum cholesterol is related to an increase in suicide or violent deaths but the basis of this possible association is under investigation (60). Therefore, further studies are needed to determine the effects of lipids on depression during menopause.

\section{Conclusions}

The present review describes the correlation between hormonal changes and depression in menopausal status. In menopause, the final menstrual stage, hormonal and neurotransmitter changes may lead to depression. Overnight cortisol levels during the menopausal transition were associated with estrone glucuronide, testosterone, and FSH levels. In addition, whole blood serotonin concentration is reduced during menopause. Furthermore, plasma BDNF concentration decreases significantly in postmenopausal stage. The available evidence suggests that transition to menopause and its changing hormonal and other serum markers are strongly associated with the depressed mood among women.

\section{Authors' Contributions}

All authors contributed to preparing some part of this manuscript. One gave its idea and the other edited as oth- ers found and collected subjects from the internet and books.

\section{References}

1. Prelevic GM, Jacobs HS. Menopause and post-menopause. Baillieres Clin Endocrinol Metab. 1997;11(2):311-40.

2. Greendale GA, Lee NP, Arriola ER. The menopause. Lancet. 1999;353(9152):571-80.

3. Al-Azzawi F, Palacios S. Hormonal changes during menopause Maturitas. 2009;63(2):135-7.

4. Harlow SD, Gass M, Hall JE, Lobo R, Maki P, Rebar RW, et al. Executive summary of the Stages of Reproductive Aging Workshop +10 : addressing the unfinished agenda of staging reproductive aging. Climacteric. 2012;15(2):105-14.

5. Rohr UD. The impact of testosterone imbalance on depression and women's health. Maturitas. 2002;41 Suppl 1:S25-46.

6. Noble RE. Depression in women. Metabolism. 2005;54(5 Suppl 1):49-52.

7. Birkhauser M. Depression, menopause and estrogens: is there a correlation? Maturitas. 2002;41 Suppl 1:S3-8.

8. Cohen LS, Soares CN, Vitonis AF, Otto MW, Harlow BL. Risk for new onset of depression during the menopausal transition: the Harvard study of moods and cycles. Arch Gen Psychiatry. 2006;63(4):385-90.

9. Farzanegi P, Ayatollahi P, Barari AR, Fakori Joybari M, Shirali S, Shojaee M, et al. Effect of Eight-Week Consumption of Purslane Extract on Peroxidane/Antioxidant Balance in Women with Type 2 Diabetes. Med Laboratory J. 2014;8(2):1-7.

10. Chashmpoosh M, Shirali S, Javidnia J. . Menopause and Depression. The 3rd Iranian International Conference on Women,s Health. 2014..

11. Higgins JPT, Green S. Cochrane handbook for systematic reviews of interventions.London: The Cochrane Collaboration; 2009.

12. Rajewska J, Rybakowski JK. Depression in premenopausal women: gonadal hormones and serotonergic system assessed by D-fenfluramine challenge test. Prog Neuropsychopharmacol Biol Psychiatry. 2003;27(4):705-9.

13. Okatani Y, Sagara Y. Amplification of nocturnal melatonin secretion in women with functional secondary amenorrhoea: relation to endogenous oestrogen concentration. Clin Endocrinol (Oxf). 1994;41(6):763-70.

14. Okatani Y, Morioka N, Hayashi K. Changes in nocturnal pineal melatonin synthesis during the perimenopausal period: relation to estrogen levels in female rats. JPineal Res.1999;27(2):65-72.

15. Woods NF, Mitchell ES, Smith-Dijulio K. Cortisol levels during the menopausal transition and early postmenopause: observations from the Seattle Midlife Women's Health Study. Menopause. 2009;16(4):708-18.

16. Woods NF, Carr MC, Tao EY, Taylor HJ, Mitchell ES. Increased urinary cortisol levels during the menopausal transition. Menopause. 2006;13(2):212-21.

17. Gonzales GF, Carrillo C. Blood serotonin levels in postmenopaus al women: effects of age and serum oestradiol levels. Maturitas. 1993;17(1):23-9.

18. Sohrabji F, Miranda RC, Toran-Allerand CD. Identification of a putative estrogen response element in the gene encoding brain-derived neurotrophic factor. Proc Natl Acad Sci U S A. 1995;92(24):11110-4.

19. Campbell S, Whitehead M. Oestrogen therapy and the menopausal syndrome. Clin Obstet Gynaecol. 1977;4(1):31-47.

20. Schneider MA, Brotherton PL, Hailes J. The effect of exogenous oestrogens on depression in menopausal women. Med J Aust. 1977;2(5):162-3.

21. Schmidt PJ, Nieman L, Danaceau MA, Tobin MB, Roca CA Murphy $\mathrm{JH}$, et al. Estrogen replacement in perimenopauserelated depression: a preliminary report. Am J Obstet Gynecol. 2000;183(2):414-20.

22. Pearlstein T, Rosen K, Stone AB. Mood disorders and menopause. Endocrinol Metab Clin North Am. 1997;26(2):279-94.

23. Huerta R, Mena A, Malacara JM, Diaz de Leon J. Symptoms at perimenopausal period: its association with attitudes toward sexual- 
ity, life-style, family function, and FSH levels. Psychoneuroendocrinology. 1995;20(2):135-48.

24. Yazici KM, Akinci A, Sutcu A, Ozcakar L. Bone mineral density in premenopausal women with major depressive disorder. Psychiatry Res. 2003;117(3):271-5.

25. Okatani Y, Morioka N, Wakatsuki A. Changes in nocturnal melatonin secretion in perimenopausal women: correlation with endogenous estrogen concentrations. J Pineal Res. 2000;28(2):111-8.

26. Reiter RJ. Pineal melatonin: cell biology of its synthesis and of its physiological interactions. Endocr Rev. 1991;12(2):151-80.

27. Luttge WG, Wallis CJ. In vitro accumulation and saturation of $3 \mathrm{H}$-progestins in selected brain regions and in the adenohypophysis, uterus and pineal of the female rat. Steroids. 1973; 22(4):493-502.

28. Cardinali DP, Nagle CA, Rosner JM. Effects of estradiol on melatonin and protein synthesis in the rat pineal organ. Horm Res. 1974;5(5):304-10.

29. Houssay AB, Barcelo AC. Effects of estrogens and progesterone upon the biosynthesis of melatonin by the pineal gland. Experientia. 1972;28(4):478-9.

30. Cardinali DP, Vacas MI, Ritta MN. A biphasic effect of estradiol on serotonin metabolism in rat pineal organ cultures. Experientia. 1981;37(2):203-4.

31. Berga SL, Mortola JF, Yen SS. Amplification of nocturnal melatonin secretion in women with functional hypothalamic amenorrhea.J Clin Endocrinol Metab. 1988;66(1):242-4.

32. Brzezinski A, Lynch HJ, Seibel MM, Deng MH, Nader TM, Wurtman RJ. The circadian rhythm of plasma melatonin during the normal menstrual cycle and in amenorrheic women. J Clin Endocrinol Metab. 1988;66(5):891-5.

33. Bartsch C, Seeger H, Muck AO, Lippert TH. The effect of estradiol on the production of melatonin in postmenopausal women. Int J Clin Pharmacol Ther. 1995;33(7):401-3.

34. McEwen BS. Physiology and neurobiology of stress and adaptation: central role of the brain. Physiol Rev. 2007;87(3):873-904.

35. Yen SS, Laughlin GA. Aging and the adrenal cortex. Exp Gerontol 1998;33(7-8):897-910.

36. Laughlin GA, Barrett-Connor E. Sexual dimorphism in the influence of advanced aging on adrenal hormone levels: the Rancho Bernardo Study. J Clin Endocrinol Metab. 2000;85(10):3561-8.

37. Vamvakopoulos NC, Chrousos GP. Evidence of direct estrogenic regulation of human corticotropin-releasing hormone gene expression. Potential implications for the sexual dimophism of the stress response and immune/inflammatory reaction. J Clin Invest. 1993;92(4):1896-902.

38. Mattsson C, Olsson T. Estrogens and glucocorticoid hormones in adipose tissue metabolism. Curr Med Chem. 2007;14(27):2918-24.

39. Dorn LD, Chrousos GP, editors. The neurobiology of stress: understanding regulation of affect during female biological transitions.; Seminars in reproductive endocrinology.; 1997; pp. 19-35.

40. Greendale GA, Unger JB, Rowe JW, Seeman TE. The relation between cortisol excretion and fractures in healthy older people: results from the MacArthur studies-Mac. J Am Geriatr Soc. 1999;47(7):799-803.

41. Worthman CM. Endocrine pathways in differential well-being across the life course. Kuh and Hardy. 2002:197-232.

42. Meneses A. 5-HT system and cognition. Neurosci Biobehav Rev. 1999;23(8):1111-25.

43. Joffe H, Cohen LS. Estrogen, serotonin, and mood disturbance: where is the therapeutic bridge? Biol Psychiatry. 1998;44(9):798-811.

44. Guicheney P, Leger D, Barrat J, Trevoux R, De Lignieres B, Roque $\mathrm{P}$, et al. Platelet serotonin content and plasma tryptophan in peri- and postmenopausal women: variations with plasma oestrogen levels and depressive symptoms. Eur J Clin Invest. 1988;18(3):297-304

45. Berendsen HH. The role of serotonin in hot flushes. Maturitas 2000;36(3):155-64

46. Rubinow DR, Schmidt PJ, Roca CA. Estrogen-serotonin interac tions: implications for affective regulation. Biol Psychiatry. 1998; 44(9):839-50.

47. Altar CA, Cai N, Bliven T, Juhasz M, Conner JM, Acheson AL, et al Anterograde transport of brain-derived neurotrophic factor and its role in the brain. Nature. 1997;389(6653):856-60.

48. Hefti F, Knusel B, Lapchak PA. Chapter 31: Protective effects of nerve growth factor and brain-derived neurotrophic factor on basal forebrain cholinergic neurons in adult rats with partial fimbrial transections. Progress in brain research. 1993;98:257-63.

49. Lindsay RM. Neurotrophic growth factors and neurodegenerative diseases: therapeutic potential of the neurotrophins and ciliary neurotrophic factor. Neurobiol Aging. 1994;15(2):249-51.

50. Begliuomini S, Casarosa E, Pluchino N, Lenzi E, Centofanti M Freschi L, et al. Influence of endogenous and exogenous sex hormones on plasma brain-derived neurotrophic factor. Hum Reprod. 2007;22(4):995-1002.

51. Tapia-Arancibia L, Rage F, Givalois L, Arancibia S. Physiology of BDNF: focus on hypothalamic function. Front Neuroendocrinol. 2004;25(2):77-107.

52. Scharfman HE, MacLusky NJ. Estrogen and brain-derived neurotrophic factor (BDNF) in hippocampus: complexity of steroid hormone-growth factor interactions in the adult CNS. Front Neuroendocrinol. 2006;27(4):415-35.

53. Kimmick GG, Lovato J, McQuellon R, Robinson E, Muss HB. Randomized, double-blind, placebo-controlled, crossover study of sertraline (Zoloft) for the treatment of hot flashes in women with early stage breast cancer taking tamoxifen. Breast $J$. 2006;12(2):114-22.

54. Loprinzi CL, Sloan JA, Perez EA, Quella SK, Stella PJ, Mailliard JA, et al. Phase III evaluation of fluoxetine for treatment of hot flashes. JClin Oncol. 2002;20(6):1578-83.

55. Puri BK. Nutrients, Stress, and Medical Disorders. United States: Springe; 2005. pp. 221-30.Lipids and Depression.

56. Kannel WB, Hjortland MC, McNamara PM, Gordon T. Menopause and risk of cardiovascular disease: the Framingham study. Ann Intern Med. 1976;85(4):447-52.

57. Stevenson JC, Crook D, Godsland IF. Influence of age and menopause on serum lipids and lipoproteins in healthy women. Ath erosclerosis. 1993;98(1):83-90.

58. Ushiroyama T, Okamoto Y, Sugimoto O. Plasma lipid and lipoprotein levels in perimenopausal women. Clinical research in 1198 Japanese women. Acta Obstet Gynecol Scand. 1993;72(6):428-33.

59. Cho EJ, Min YJ, Oh MS, Kwon JE, Kim JE, Lee WS, et al. Effects of the transition from premenopause to postmenopause on lipids and lipoproteins: quantification and related parameters. Korean JIntern Med. 2011;26(1):47-53.

60. Manfredini R, Caracciolo S, Salmi R, Boari B, Tomelli A, Gallerani M. The association of low serum cholesterol with depression and suicidal behaviours: new hypotheses for the missing link. J Int Med Res. 2000;28(6):247-57. 